\title{
Mostafa GabBOUHY ZOUBIDA MGHAZLI \\ Un résultat d'existence de solution faible d'un système parabolique-elliptique non linéaire doublement dégénéré
}

\author{
Annales de la faculté des sciences de Toulouse $6^{e}$ série, tome 10, \\ $\mathrm{n}^{\circ} 3$ (2001), p. 533-546 \\ <http://www.numdam.org/item?id=AFST_2001_6_10_3_533_0>
}

(C) Université Paul Sabatier, 2001, tous droits réservés.

L'accès aux archives de la revue «Annales de la faculté des sciences de Toulouse » (http://picard.ups-tlse.fr/ annales/) implique l'accord avec les conditions générales d'utilisation (http://www.numdam.org/conditions). Toute utilisation commerciale ou impression systématique est constitutive d'une infraction pénale. Toute copie ou impression de ce fichier doit contenir la présente mention de copyright.

\section{Numdam}

Article numérisé dans le cadre du programme Numérisation de documents anciens mathématiques http://www.numdam.org/ 


\title{
Un résultat d'existence de solution faible d'un système parabolique-elliptique non linéaire doublement dégénéré(*)
}

\author{
Mostafa Gabbouhy, Zoubida MghaZli ${ }^{(1)}$
}

\begin{abstract}
RÉSUMÉ. - Dans cet article, nous montrons un théorème d'existence de solution faible des équations modélisant l'écoulement diphasique incompressible eau-air dans un milieu poreux non saturé. Ce système d'équations est de type parabolique-elliptique nonlinéaire, doublement dégénéré et fortement couplé. Le théorème d'éxistence est démontré en deux étapes. Dans la première nous introduisons une régularisation de type parabolique. L'existence d'une solution $u_{\epsilon}$ de la formulation régularisée est obtenue par application des résultats de Alt \& Luckhauss [1]. La deuxième étape consiste à montrer que sa limite est solution du problème dégénéré.
\end{abstract}

Abstract. - We consider here a two-phase incompressible flow model in unsaturated porous media. This flow is governed by a nonlinear, degenerate, singular and strongly coupled parabolic-elliptic system. We give an existence result of weak solution in two steps. In the first one we introduce a regularisation technique of parabolic type. To show the existence of the regularized problem solution, we use Alt \& Luckhauss [1] technique.In the second step we extract the solution as a limit of solutions of the regularized problem.

\section{Introduction}

La modélisation d'écoulement diphasique incompressible eau-air dans un milieu poreux non saturé conduit au système parabolique-elliptique non

(*) Reçu le 2 août 1999, accepté le 22 novembre 2001

(1) Laboratoire SIANO, Dép. de Maths et d'Informatique, Faculté des Sciences, Université Ibn Tofaïl, BP 133, Kénitra, Maroc.

e-mail: mghazli_zoubida@yahoo.com, gabbouhy@hotmail.com Ce travail a été mené dans le cadre de l'Action Intégrée 844/95 et du programme PARS No MI019. 
linéaire doublement dégénéré suivant: (voir $[3,11]$ pour plus de détails sur le modèle)

$$
\begin{aligned}
-\frac{\partial \theta(u)}{\partial t}-\operatorname{div}\left(k_{w}(\theta(u)) \nabla u-k_{w}(\theta(u)) \nabla v\right) & =0, & & \text { dans } Q_{T} \\
-\operatorname{div}\left(k(\theta(u)) \nabla v-k_{w}(\theta(u)) \nabla u\right) & =0, & & \text { dans } Q_{T}
\end{aligned}
$$

où $\Omega \subset \mathbb{R}^{d}, d=1,2,3$ représente le domaine d'écoulement de frontière $\Gamma=\partial \Omega=\bar{\Gamma}_{N} \cup \bar{\Gamma}_{D} ;[0, T]$ représente l'intervalle de temps $(T>0)$. On note par $Q_{T}$ le cylindre $\left.\Omega \times\right] 0, T$. Les inconnues de ce système sont la pression capillaire $u$ et la pression de la phase air $v$. Les coefficients du système sont la teneur en eau $\theta=\theta(u)$, la conductivité hydraulique de la phase eau $k_{w}(\theta(u))$ et la conductivité totale $k(\theta(u))=k_{w}(\theta(u))+k_{a}(\theta(u))$, où $k_{a}(\theta(u))$ est la conductivité hydraulique de la phase air. Nous notons que le système (1.1), (1.2) est doublement dégénéré du fait que les termes de diffusion $k_{w}$ et $k_{a}$, ainsi que la dérivée temporelle $\partial \theta(u) / \partial t$ s'annulent en certaines valeurs de $u$.

Nous appelons problème $(\mathrm{P})$, le système (1.1)-(1.2) muni des conditions initiales et aux limites suivantes,

$$
\begin{array}{rc}
\theta(u(x, 0))=\theta_{0}(x), & \text { dans } \Omega \\
u=u_{D}, & \text { sur } \left.\Gamma_{D} \times\right] 0, T[ \\
v=v_{D}, & \text { sur } \left.\Gamma_{D} \times\right] 0, T[ \\
\left(k_{w}(\theta(u)) \nabla u-k_{w}(\theta(u)) \nabla v\right) . \nu=0, & \text { sur } \left.\Gamma_{N} \times\right] 0, T[ \\
\left(k(\theta(u)) \nabla v-k_{w}(\theta(u)) \nabla u\right) . \nu=0, & \text { sur } \left.\Gamma_{N} \times\right] 0, T[
\end{array}
$$

et tel que $u$ vérifie,

$$
0 \leqslant \delta \leqslant u \leqslant u_{M}, \quad \text { p.p. }(x, t) \in Q_{T}
$$

Alt et Luckhauss [1] ont étudié une famille de systèmes paraboliqueselliptiques satisfaisant la condition d'ellipticité et ont donné un théorème d'existence de solution faible. Kroener et Luckhauss [10] ont traité le système d'écoulement diphasique incompressible huile-eau écrit sous la forme:

$$
\begin{aligned}
& \frac{\partial s_{1}}{\partial t}-\operatorname{div}\left(K_{1}\left(s_{1}\right)\left(\nabla p_{1}+e_{1}\right)\right)=0, \quad \text { dans } Q_{T} \\
& \frac{\partial s_{2}}{\partial t}-\operatorname{div}\left(K_{2}\left(s_{1}\right)\left(\nabla p_{2}+e_{2}\right)\right)=0, \quad \text { dans } Q_{T}
\end{aligned}
$$

où $s_{i}, p_{i}, i=1,2$ représentent respectivement la saturation et la pression de la phase $i$, l'indice 1 (resp. 2) représente la phase huile (resp. la phase eau). 
Cependant, les auteurs ont écarté toute dégénérescence ou singularité dans les équations ci-dessus et ont montré, en appliquant directement le résultat de [1], l'existence d'une solution faible du système (1.9), (1.10) muni de conditions initiales sur $s_{i}$ et de conditions aux limites mêlées de type Dirichlet sur une partie de la frontière et Neumann sur l'autre. Du fait du fort couplage de ce dernier système, Chavent et Jaffré [7], Antonsev et al. [4] l'ont reformulé, via la notion de pression globale, de manière à affaiblir le couplage entre les deux équations et rendre l'analyse plus aisée. Le système résultant est formé d'une équation parabolique en saturation et d'une équation elliptique en pression. Dans $[4,5,2,9,8]$, on trouve l'analyse mathématique d'un tel système et d'autres modèles liés à l'ingénierie pétrolière.

Dans cet article, nous nous intéressons au domaine de l'hydrologie qui nécessite une bonne compréhension des écoulements en milieux poreux nonsaturés. Nous allons donner un résultat d'existence pour le modèle (1.1)(1.8). Le choix des inconnues a été motivé par l'intérêt physique (cf. [11], [3]). Les difficultés principales de notre problème sont le fort couplage des équations, la double dégénérescence et la double nonlinéarité. Ce résultat a été obtenu à l'aide de la technique de régularisation qui consiste à résoudre tout d'abord la suite de problèmes régularisés suivants,

$$
\begin{array}{r}
\delta \leqslant u_{\epsilon} \leqslant u_{M}, \text { p.p. dans } Q_{T}(1.11) \\
-\frac{\partial \theta\left(u_{\epsilon}\right)}{\partial t}-\operatorname{div}\left(k_{w}\left(\theta\left(u_{\epsilon}\right)\right) \nabla u_{\epsilon}-k_{w}\left(\theta\left(u_{\epsilon}\right)\right) \nabla v_{\epsilon}\right)-\epsilon \Delta u_{\epsilon}=0, \text { dans } Q_{T}(1.12) \\
-\operatorname{div}\left(k\left(\theta\left(u_{\epsilon}\right)\right) \nabla v_{\epsilon}-k_{w}\left(\theta\left(u_{\epsilon}\right)\right) \nabla u_{\epsilon}\right)=0, \text { dans } Q_{T}(1.13) \\
\theta\left(u_{\epsilon}(x, 0)\right)=\theta_{0}(x), \text { dans } \Omega(1.14) \\
\left.u_{\epsilon}=u_{D}, \text { sur } \Gamma_{D} \times\right] 0 . T[(1.15) \\
\left.v_{\epsilon}=v_{D}, \text { sur } \Gamma_{D} \times\right] 0 . T[(1.16) \\
\left.\left(k_{w}\left(\theta\left(u_{\epsilon}\right)\right) \nabla u_{\epsilon}-k_{w}\left(\theta\left(u_{\epsilon}\right)\right) \nabla v_{\epsilon}\right) . \nu+\epsilon \frac{\partial u_{\epsilon}}{\partial \nu}=0, \text { sur } \Gamma_{N} \times\right] 0 . T[(1.17) \\
\left.\left(k\left(\theta\left(u_{\epsilon}\right)\right) \nabla v_{\epsilon}-k_{w}(\theta(u)) \nabla u_{\epsilon}\right) . \nu=0, \text { sur } \Gamma_{N} \times\right] 0 . T[(1.18)
\end{array}
$$

puis à montrer que la suite, définie comme solution faible du problème perturbé, converge et que sa limite est solution du problème dégénéré.

La suite de cet article est organisée de la manière suivante. Danss le second paragraphe, nous précisons les hypothèses sur le domaine d'écoulcunent et sur les coefficients du système parabolique-elliptique (1.1)-(1.8). Nous donnons ensuite le résultat d'existence d'une solution faible du problème régularisé ainsi que les étapes principales de la démonstration de ce résult at. L'objet du dernier paragraphe est de montrer que le problème dégénéré initial admet une solution faible. 


\section{Hypothèses et étude du problème régularisé}

Dans ce paragraphe, nous allons préciser les hypothèses dont on aura besoin par la suite. Nous signalons que la majorité de ces hypothèses sont physiques. Puis nous allons définir la formulation variationnelle du problème régularisé et nous donnons un résultat d'existence de solution faible de ce problème.

\subsection{Hypothèses sur les données}

$\left(\mathbf{H}_{1}\right) \Omega$ est un ouvert borné de $\mathbb{R}^{d}$, avec $d=1,2,3$, de frontière $\Gamma=\bar{\Gamma}_{D} \cup \bar{\Gamma}_{N}$ suffisamment régulière telle que $\operatorname{mes}\left(\Gamma_{D}\right)>0$. On suppose par ailleurs qu'il existe $\delta$ strictement positif tel que $\delta \leqslant u_{D} \leqslant u_{M}$ p.p. dans $Q_{T}$ et que $u_{D}, v_{D} \in L^{2}\left(0, T ; H^{1}(\Omega)\right)$.

$\left(\mathbf{H}_{2}\right)$ la fonction $\theta:\left[0, u_{M}\right] \rightarrow \mathbb{R}$ est continue, décroissante et sa dérivée $\theta^{\prime}(u)$ s'annule en 0 et $u_{M}$ et $\left.\theta^{\prime}(u)<0, \forall u \in\right] 0, u_{M}[$.

$\left(\mathbf{H}_{3}\right) k_{w}$ et $k_{a}=k-k_{w}$ sont deux fonctions positives et continues de $\left[\theta_{r}, \theta_{s}\right] \rightarrow \mathbb{R}$, telles que $k_{w}\left(\theta_{r}\right)=k_{a}\left(\theta_{s}\right)=0$ et $k_{w}$ (resp. $k_{a}$ ) est strictement positive sur $\left.] \theta_{r}, \theta_{s}\right]$ (resp. sur $\left[\theta_{r}, \theta_{s}[)\right.$, où $\theta_{r}=\theta\left(u_{M}\right)$ et $\theta_{s}=\theta(0)$.

$\left(\mathbf{H}_{4}\right) \theta^{0} \in L^{\infty}(\Omega)$ et Il existe $u^{0} \in L^{\infty}(\Omega)$ tel que: $\theta^{0}(x)=\theta\left(u^{0}(x)\right)$ avec $\delta \leqslant u^{0}(x) \leqslant u_{M}$, p.p. $x \in \Omega$

Remarque. - L'hypothèse $\left(\mathrm{H}_{3}\right)$ implique qu'il existe $\alpha_{*}>0$ tel que : $k(t)>\alpha_{*}>0, \forall t \in\left[\theta_{r}, \theta_{s}\right]$

\subsection{Formulation variationnelle du problème régularisé}

On considère l'espace de Hilbert $V$ défini par:

$$
V=\left\{\varphi \in H^{1}(\Omega) ; \varphi=0 \text { sur } \Gamma_{D}\right\}
$$

muni du produit scalaire $(\varphi, \psi)_{V}=\int_{\Omega} \nabla \varphi \nabla \psi d x$. On note par $\|$. \| la norme associée à ce produit scalaire et par $V^{\prime}$ le dual de $V$. Posons

$$
\begin{aligned}
& K_{1}=\left\{\varphi \in H^{1}(\Omega), \varphi=u_{D} \text { sur } \Gamma_{D}\right\} \\
& K_{2}=\left\{\varphi \in H^{1}(\Omega), \varphi=v_{D} \text { sur } \Gamma_{D}\right\}
\end{aligned}
$$

Notons par $k_{w}^{\epsilon}$ la fonction $k_{w}^{\epsilon}\left(\theta\left(u_{\epsilon}\right)\right)=k_{w}\left(\theta\left(u_{\epsilon}\right)\right)+\epsilon$. 
Définition du problème régularisé $\left(\mathbf{P}_{\epsilon}\right)$ :

Trouver $\left(u_{\epsilon}, v_{\epsilon}\right)$ tels que:

(i)

$$
\delta \leqslant u_{\epsilon} \leqslant u_{M}, \text { p.p. dans } Q_{T}
$$

(ii)

$$
u_{\epsilon} \in u_{D}+L^{2}(0, T ; V), \frac{\partial \theta\left(u_{\epsilon}\right)}{\partial t} \in L^{2}\left(0, T ; V^{\prime}\right)
$$

(iii)

$$
v_{\epsilon} \in v_{D}+L^{2}(0, T ; V)
$$

(iv)

$$
\begin{aligned}
-\int_{0}^{T}< & <\frac{\partial \theta\left(u_{\epsilon}\right)}{\partial t}, \varphi>d t=\int_{0}^{T} \int_{\Omega} \theta\left(u_{\epsilon}\right) \frac{\partial \varphi}{\partial t} d x d t \\
& +\int_{\Omega} \theta_{0}(x) \varphi(x, 0) d x, \forall \varphi \in \mathcal{D}(\Omega \times[0, T[)
\end{aligned}
$$

(v)

$$
\begin{gathered}
-\int_{0}^{T}<\frac{\partial \theta\left(u_{\epsilon}\right)}{\partial t}, w>d t+\int_{0}^{T} \int_{\Omega}\left(k_{w}^{\epsilon}\left(\theta\left(u_{\epsilon}\right)\right) \nabla u_{\epsilon}\right. \\
\left.-k_{w}\left(\theta\left(u_{\epsilon}\right)\right) \nabla v_{\epsilon}\right) \nabla w d x d t=0, \forall w \in L^{2}(0, T ; V)
\end{gathered}
$$

(vi)

$$
\int_{0}^{T} \int_{\Omega}\left(k\left(\theta\left(u_{\epsilon}\right)\right) \nabla v_{\epsilon}-k_{w}\left(\theta\left(u_{\epsilon}\right)\right) \nabla u_{\epsilon}\right) \nabla \psi d x d t=0, \quad \forall \psi \in L^{2}(0, T ; V)
$$

Dans le reste de ce paragraphe, nous allons omettre l'indice $\epsilon$ pour simplifier la présentation.

Proposition 2.1. - Sous les hypothèses $\left(H_{1}\right)-\left(H_{4}\right)$. le problème régularisé $\left(P_{\epsilon}\right)$ admet au moins une solution.

Esquisse de la démonstration. - La démontration de ce résultat suit de près celle donnée par Alt \& Luckhauss [1]. En effet, nous introduisons le problème semi-discrétisé $\left(\mathrm{P}_{\epsilon, s}\right)$ en effectuant une discrétisation en temps du problème $\left(\mathrm{P}_{\epsilon}\right)$ : 
Trouver $u^{n} \in K_{1}$ telle que $\delta \leqslant u^{n} \leqslant u_{M}$, p.p. $x \in \Omega$ et $v^{n} \in K_{2}$ vérifiant les équations suivantes:

$$
\begin{gathered}
-\int_{\Omega} \frac{\theta\left(u^{n}\right)-\theta\left(u^{n-1}\right)}{\Delta t} w d x+ \\
\int_{\Omega}\left(k_{w}^{\epsilon}\left(\theta\left(u^{n}\right)\right) \nabla u^{n}-k_{w}\left(\theta\left(u^{n}\right)\right) \nabla v^{n}\right) \nabla w d x=0, \quad \forall w \in V \\
\int_{\Omega}\left(k\left(\theta\left(u^{n}\right)\right) \nabla v^{n}-k_{w}\left(\theta\left(u^{n}\right)\right) \nabla u^{n}\right) \nabla \psi d x=0, \quad \forall \psi \in V
\end{gathered}
$$

où $\Delta t=\frac{T}{N}, N \in \mathbb{N}^{*}$, représente le pas de temps. On note $t_{n}=n . \Delta t$ pour $n=0, \ldots, N$ et $\chi_{\left.t^{n-1}, t^{n}\right]}$ la fonction caractéristique de l'intervalle $\left.] t^{n-1}, t^{n}\right]$ et on définit les fonctions constantes par morceaux sur $] 0, T[$ suivantes:

$$
\begin{aligned}
& u_{\Delta t}(x, 0)=u_{0}(x) \\
& u_{\Delta t}(x, t)=\sum_{n=1}^{N} u^{n}(x) \chi_{] t^{n-1}, t^{n}\right]}(t) \\
& v_{\Delta t}(x, t)=\sum_{n=1}^{N} v^{n}(x) \chi_{] t^{n-1}, t^{n}\right]}(t) \\
& \theta_{\Delta t}(x, t)=\sum_{n=1}^{N} \frac{\theta\left(u^{n}(x)\right)-\theta\left(u^{n-1}(x)\right)}{\Delta t} \chi_{] t^{n-1}, t^{n}\right]}(t)
\end{aligned}
$$

Nous nous proposons de montrer que la suite $\left(u_{\Delta t}, v_{\Delta t}\right)$ converge et que sa limite est solution du problème régularisé. Les étapes principales de la démonstration sont données dans les quatre lemmes suivants:

Lemme 2.1. - Le problème semi-discrétisé $\left(P_{\epsilon, s}\right)$ admet une solution $\left(u^{n}, v^{n}\right)$.

La démonstration de ce lemme est basée sur le fait que l'opérateur régularisé du second ordre du système $(2.7)-(2.8)$

$$
A(z)=\left(\begin{array}{cc}
k_{w}(\theta(z))+\epsilon & -k_{w}(\theta(z)) \\
-k_{w}(\theta(z)) & k_{w}(\theta(z))+k_{a}(\theta(z))
\end{array}\right)
$$

est elliptique. Le théorème du point fixe de Schauder-Tychonov permet de conclure le résultat.

Lemme 2.2. - Sous les hypothèses $\left(H_{1}\right)-\left(H_{4}\right)$, les suites $u_{\Delta t}$ et $v_{\Delta t}$ données par (2.7)-(2.11) vérifient les estimations suivantes:

$$
\sup _{0 \leqslant t \leqslant T} \int_{\Omega} \Psi\left(u_{\Delta t}\right) d x+\epsilon \int_{0}^{T}\left\|u_{\Delta t}\right\|^{2} d t \leqslant C
$$


Un résultat d'existence de solution faible

$$
\epsilon \int_{0}^{T}\left\|v_{\Delta t}\right\|^{2} d t \leqslant C
$$

où $\Psi(z)=-\theta(z) . z+\int_{0}^{z} \theta(s) d s$ et $C$ est une constante indépendante de $\Delta t$.

Lemme 2.3. - La suite $u_{\Delta t}$ vérifie l'estimation suivante:

$$
\begin{array}{r}
-\int_{0}^{T-h} \int_{\Omega}\left(\theta\left(u_{\Delta t}(t+h)\right)-\theta\left(u_{\Delta t}(t)\right)\right)\left(u_{\Delta t}(t+h)-u_{\Delta t}(t)\right) d x d t \leqslant C h, \\
\forall h \in] 0, T[
\end{array}
$$

où $C$ est une constante indépendante de $\Delta t$ et de $h$.

Lemme 2.4. - Soient $\left(u_{k}\right)$ une suite de $L^{2}\left(0, T ; H^{1}(\Omega)\right)$, vérifiant $\delta \leqslant$ $u_{k} \leqslant u_{M}$, p.p. dans $Q_{T}$, qui converge faiblement vers $u$ dans $L^{2}\left(0, T ; H^{1}(\Omega)\right)$. Soient $\theta$ une fonction vérifiant l'hypothèse $\left(H_{2}\right)$ et $C$ une constante indépendante de $k$ telles que:

$$
\begin{array}{r}
-\int_{0}^{T-h} \int_{\Omega}\left(\theta\left(u_{k}(t+h)\right)-\theta\left(u_{k}(t)\right)\right)\left(u_{k}(t+h)-u_{k}(t)\right) d x d t \leqslant C h, \\
\text { pour tout } h \in] 0, T[
\end{array}
$$

Alors on peut extraire une sous-suite, notée encore $u_{k}$, telle que:

$$
\theta\left(u_{k}\right) \rightarrow \theta(u) \text { dans } L^{p}\left(Q_{T}\right) \text { fort, } \forall p \in\left[1,+\infty\left[\text {, et p.p. dans } Q_{T}\right.\right.
$$

Remarque. - Du fait que la fonction $\theta^{-1}$ est continue et bornée, nous obtenons, à l'aide du théorème de Lebesgue, le résultat suivant

$$
u_{\Delta t} \rightarrow u \text { dans } L^{p}\left(Q_{T}\right) \text { fort, } \forall p \in\left[1,+\infty\left[\text {, et p.p. dans } Q_{T}\right.\right.
$$

En multipliant les équations (2.7)-(2.8) par $\Delta t$, et en sommant sur $n$ de 0 à $N$, on trouve,

$$
\begin{gathered}
-\int_{0}^{T} \int_{\Omega} \theta_{\Delta t} w d x d t+\int_{0}^{T} \int_{\Omega} k_{w}^{\epsilon}\left(\theta\left(u_{\Delta t}\right)\right) \nabla u_{\Delta t} \nabla w d x d t- \\
-\int_{0}^{T} \int_{\Omega} k_{w}\left(\theta\left(u_{\Delta t}\right)\right) \nabla v_{\Delta t} \nabla w d x d t=0, \forall w \in V \\
\int_{0}^{T} \int_{\Omega} k\left(\theta\left(u_{\Delta t}\right)\right) \nabla v_{\Delta t} \nabla \psi d x d t-\int_{0}^{T} \int_{\Omega} k_{w}\left(\theta\left(u_{\Delta t}\right)\right) \nabla u_{\Delta t} \nabla \psi d x d t=0, \forall \psi \in V
\end{gathered}
$$


L'équation (2.19) et le lemme 2.2 permettent d'avoir:

$$
\left\|\theta_{\Delta t}\right\|_{L^{2}\left(0, T ; V^{\prime}\right)} \leqslant C
$$

En vertu de (2.13), (2.14), (2.18) et (2.21), nous en déduisons:

$$
\begin{array}{rr}
u_{\Delta t} \rightarrow u, & \text { faiblement dans } L^{2}(0, T ; V) \\
v_{\Delta t} \rightarrow v, & \text { faiblement dans } L^{2}(0, T ; V) \\
\theta_{\Delta t} \rightarrow \frac{\partial \theta}{\partial t}(u), & \text { faiblement dans } L^{2}\left(0, T ; V^{\prime}\right) \\
k_{w}\left(\theta\left(u_{\Delta t}\right)\right) \rightarrow k_{w}(\theta(u)), & \text { fortement dans } L^{2}\left(Q_{T}\right) \\
k\left(\theta\left(u_{\Delta t}\right)\right) \rightarrow k(\theta(u)), & \text { fortement dans } L^{2}\left(Q_{T}\right)
\end{array}
$$

Donc, en passant à la limite dans les équations (2.19)-(2.20), on en déduit que $(u, v)$ est solution faible du problème régularisé.

\section{Etude du problème dégénéré}

Dans ce paragraphe, nous allons montrer que la suite $u_{\epsilon}$, solution du problème régularisé, converge et que sa limite est solution du problème dégénéré. Afin d'absorber la dégénérescence, nous introduisons la fonction $\phi(s)=\int_{0}^{s} \sqrt{k_{w}(\theta(\zeta))} d \zeta$. D'après l'hypothèse $\left(\mathrm{H}_{3}\right)$, nous en déduisons que $\phi$ est strictement croissante et de classe $\mathcal{C}^{1}$ sur $\left[0, u_{M}\right]$. En utilisant la fonction $\phi$, nous introduisons ci-dessous la définition d'une solution faible du problème dégénéré.

\subsection{Formulation variationnelle du problème dégénéré}

Définition du problème dégénéré $\left(P_{d}\right)$ :

Trouver $(u, v)$ tel que:

(i) $\delta \leqslant u \leqslant u_{M}$, p.p. dans $Q_{T}$

(ii) $\phi(u) \in L^{2}\left(0, T ; H^{1}(\Omega)\right)$ tel que $\gamma \phi(u)=\phi\left(u_{D}\right)$ sur $\Gamma_{D}, \frac{\partial \theta(u)}{\partial t} \in$ $L^{2}\left(0, T ; V^{\prime}\right)$, et $v \in v_{D}+L^{2}(0, T ; V)$

(iii) $-\int_{0}^{T}<\frac{\partial \theta(u)}{\partial t}, \varphi>d t=\int_{0}^{T} \int_{\Omega} \theta(u) \frac{\partial \varphi}{\partial t} d x d t+\int_{\Omega} \theta_{0}(x) \varphi(x, 0) d x, \quad \forall \varphi \epsilon$ $\mathcal{D}(\Omega \times[0, T[)$

(iv) $-\int_{0}^{T}<\frac{\partial \theta(u)}{\partial t}, w>d t+\int_{0}^{T} \int_{\Omega}\left(\sqrt{k_{w}(\theta(u))} \nabla \phi(u)-k_{w}(\theta(u)) \nabla v\right) \nabla w d x d t=$ $0, \forall w \in L^{2}(0, T ; V)$

(v) $\int_{0}^{T} \int_{\Omega}\left(k(\theta(u)) \nabla v-\sqrt{k_{w}(\theta(u))} \nabla \phi(u)\right) \nabla \psi d x d t=0, \quad \forall \psi \in L^{2}(0, T ; V)$ 


\subsection{Résultat d'existence pour le problème dégénéré $\left(P_{\mathrm{d}}\right)$}

Proposition 3.1. - Sous les hypothèses $\left(H_{1}\right)-\left(H_{4}\right)$, le problème $\left(P_{\mathrm{d}}\right)$ admet au moins une solution.

Avant de montrer ce résultat, nous allons donner quelques lemmes utiles pour la démonstration.

Lemme 3.1. - Soient $\theta$ une fonction vérifiant $\left(H_{2}\right), u \in L^{2}\left(0, T ; H^{1}(\Omega)\right)$ tel que $\delta \leqslant u \leqslant u_{M}$, p.p. dans $Q_{T}, \frac{\partial \theta}{\partial t}(u) \in L^{2}\left(0, T ; V^{\prime}\right)$ et

$$
\begin{array}{r}
-\int_{0}^{T}<\frac{\partial \theta}{\partial t}(u), \varphi>d t=\int_{0}^{T} \int_{\Omega} \theta(u) \frac{\partial \varphi}{\partial t} d x d t+\int_{\Omega} \theta_{0}(x) \varphi(x, 0) d x, \\
\forall \varphi \in \mathcal{D}(\Omega \times[0, T[)
\end{array}
$$

Alors,

(i) l'application $s \rightarrow \int_{\Omega} \Psi_{d}(u(x, s)) d x$ est continue sur $[0, T]$,

$$
\text { ò̀ } \Psi_{d}(z)=\int_{\theta_{0}(z)}^{\theta(z)}\left(\theta^{-1}(s)-u_{D}\right) d s, \quad \forall z \in\left[\delta, u_{M}\right]
$$

(ii) pour tout $s \in[0, T]$, nous avons:

$$
-\int_{0}^{s}<\frac{\partial \theta}{\partial t}(u), u-u_{D}>d t=\int_{\Omega} \Psi_{d}(u(x, s)) d x
$$

La démonstration de ce lemme est donnée dans [9].

Lemme 3.2. - Soient $X$ un espace de Hilbert muni de la norme $\|\cdot\|_{X}$. et $f$ un élément de $L^{2}(0, T ; X)$ tel que $\frac{\partial f}{\partial t} \in L^{2}(0, T ; X)$.

Nous avons l'inégalité suivante,

$$
\int_{0}^{T-h}\|f(t+h)-f(t)\|_{X}^{2} d t \leqslant h^{2} \int_{0}^{T}\left\|\frac{\partial f}{\partial t}\right\|_{X}^{2} d t, \quad \forall h \in[0, T]
$$

La démonstration de ce lemme est donnée dans [6].

LEMME 3.3. - Soient $\theta$ une fonction vérifiant $\left(H_{2}\right), \phi$ une fonction de classe $\mathcal{C}^{1}$ sur $\left[0, u_{M}\right]$ à valeurs dans $\mathbb{R}, u_{\epsilon}$ une suite de $L^{2}\left(Q_{T}\right)$ et $C$ une 
constante indépendante de $\epsilon$ telles que,

$$
\begin{array}{r}
\phi\left(u_{\epsilon}\right) \in L^{2}\left(0, T ; H^{1}(\Omega)\right) \\
\frac{\partial \theta}{\partial t}\left(u_{\epsilon}\right) \in L^{2}\left(0, T ; V^{\prime}\right) \\
\left\|\nabla \phi\left(u_{\epsilon}\right)\right\|_{L^{2}\left(0, T ; L^{2}(\Omega)\right)}^{2} \leqslant C \\
\left\|\frac{\partial \theta}{\partial t}\left(u_{\epsilon}\right)\right\|_{L^{2}\left(0, T ; V^{\prime}\right)}^{2} \leqslant C
\end{array}
$$

Alors on $a$,

$$
\begin{gathered}
-\int_{0}^{T-h} \int_{\Omega}\left(\theta\left(u_{\epsilon}(t+h)\right)-\theta\left(u_{\epsilon}(t)\right)\right)\left(\phi\left(u_{\epsilon}(t+h)\right)-\phi\left(u_{\epsilon}(t)\right)\right) d x d t \\
\leqslant 2 C . h, \quad \forall h \in] 0, T[
\end{gathered}
$$

Démonstration du lemme 3.2. - Soient $h \in] 0, T\left[, v \in L^{2}(0, T ; V)\right.$, nous avons:

$$
\begin{aligned}
\int_{0}^{T-h} \int_{\Omega}\left(\theta\left(u_{\epsilon}(t+h)\right)-\right. & \left.\theta\left(u_{\epsilon}(t)\right)\right) v(t) d x d t \\
& \leqslant \int_{0}^{T-h}\left\|\theta\left(u_{\epsilon}(t+h)\right)-\theta\left(u_{\epsilon}(t)\right)\right\|_{V^{\prime}}\|v(t)\|_{V} d t \\
& \leqslant\|v\|_{L^{2}(0, T-h ; V)}\left(h^{2} \int_{0}^{T-h}\left\|\frac{\partial \theta}{\partial t}\left(u_{\epsilon}\right)\right\|_{V^{\prime}}^{2} d t\right)^{1 / 2} \\
& \leqslant h\|v\|_{L^{2}(0, T-h ; V)}\left\|\frac{\partial \theta}{\partial t}\left(u_{\epsilon}\right)\right\|_{L^{2}\left(0, T ; V^{\prime}\right)}
\end{aligned}
$$

la deuxième inégalité est obtenue en appliquant le lemme 3.3.

Pour $v=\phi\left(u_{\epsilon}(t+h)\right)-\phi\left(u_{\epsilon}(t)\right) \in V$ et compte tenu des estimations (3.1) et (3.2), on obtient l'estimation (3.3).

Démonstration de la proposition 3.1. - Pour pouvoir passer à la limite sur $\epsilon$, considérons le problème régularisé $\left(\mathrm{P}_{\epsilon}\right)$ défini par (2.1)-(2.6). Nous devons obtenir des estimations a priori indépendantes de $\epsilon$.

Les équations $(2.5)$ et $(2.6)$ de $\left(\mathrm{P}_{\epsilon}\right)$ peuvent tout d'abord se mettre sous la forme vectorielle suivante,

$$
\begin{aligned}
& \int_{0}^{T}\left(\begin{array}{ll}
-\frac{\partial \theta}{\partial t}\left(u_{\epsilon}\right) & 0
\end{array}\right) \cdot\left(\begin{array}{c}
\varphi \\
\psi
\end{array}\right)+\int_{Q_{T}}\left(\begin{array}{ll}
\nabla u_{\epsilon} & \nabla v_{\epsilon}
\end{array}\right)\left(\begin{array}{cc}
a & -a \\
-a & a+b
\end{array}\right)\left(\begin{array}{c}
\nabla \varphi \\
\nabla \psi
\end{array}\right)= \\
& =-\int_{Q_{T}}\left(\begin{array}{ll}
\nabla u_{\epsilon} & \nabla v_{\epsilon}
\end{array}\right)\left(\begin{array}{cc}
\epsilon & 0 \\
0 & 0
\end{array}\right)\left(\begin{array}{c}
\nabla \varphi \\
\nabla \psi
\end{array}\right), \quad \forall(\varphi, \psi) \in V^{2}
\end{aligned}
$$

où $a=k_{w}\left(\theta\left(u_{\epsilon}\right)\right), b=k_{a}\left(\theta\left(u_{\epsilon}\right)\right)$ et $a+b=k\left(\theta\left(u_{\epsilon}\right)\right)$. 
Prenons comme fonctions tests $\varphi=u_{\epsilon}-u_{D}, \psi=v_{\epsilon}-v_{D}$, on trouve,

$$
\begin{aligned}
& -\int_{0}^{T}<\frac{\partial \theta}{\partial t}\left(u_{\epsilon}\right), u_{\epsilon}-u_{D}>d t+\int_{Q_{T}}\left(\begin{array}{ll}
\nabla u_{\epsilon} & \nabla v_{\epsilon}
\end{array}\right) \cdot\left(\begin{array}{cc}
a & -a \\
-a & a+b
\end{array}\right)\left(\begin{array}{c}
\nabla u_{\epsilon} \\
\nabla v_{\epsilon}
\end{array}\right)+ \\
& +\epsilon \int_{Q_{T}}\left|\nabla u_{\epsilon}\right|^{2}=\int_{Q_{T}}\left(\begin{array}{ll}
\nabla u_{\epsilon} & \nabla v_{\epsilon}
\end{array}\right) \cdot\left(\begin{array}{cc}
a+\epsilon & -a \\
-a & a+b
\end{array}\right)\left(\begin{array}{c}
\nabla u_{D} \\
\nabla v_{D}
\end{array}\right)
\end{aligned}
$$

Comme la quantité ${ }^{t} X A X$, où $X=\left(\begin{array}{ll}x & y\end{array}\right)^{t}$ et $A=\left(\begin{array}{cc}a & -a \\ -a & a+b\end{array}\right)$, peut se devolopper de la façon suivante,

$$
{ }^{t} X A X=a|x-y|^{2}+b y^{2}
$$

nous avons,

$$
\begin{aligned}
& -\int_{0}^{T}<\frac{\partial \theta}{\partial t}\left(u_{\epsilon}\right), u_{\epsilon}-u_{D}>d t+\int_{Q_{T}} a\left|\nabla v_{\epsilon}-\nabla u_{\epsilon}\right|^{2}+\int_{Q_{T}} b\left|\nabla v_{\epsilon}\right|^{2}+\int_{Q_{T}} \epsilon\left|\nabla u_{\epsilon}\right|^{2}= \\
& =\int_{Q_{T}}(a+\epsilon) \nabla u_{\epsilon} \nabla u_{D}-\int_{Q_{T}} a \nabla v_{\epsilon} \nabla u_{D}-\int_{Q_{T}} a \nabla u_{\epsilon} \nabla v_{D}+\int_{Q_{T}}(a+b) \nabla v_{\epsilon} \nabla v_{D}
\end{aligned}
$$

ce qui implique

$$
\begin{aligned}
& \int_{Q_{T}} a\left|\nabla v_{\epsilon}-\nabla u_{\epsilon}\right|^{2}+\int_{Q_{T}} b\left|\nabla v_{\epsilon}\right|^{2}+\int_{Q_{T}} \epsilon\left|\nabla u_{\epsilon}\right|^{2}=\int_{0}^{T}<\frac{\partial \theta}{\partial t}\left(u_{\epsilon}\right), u_{\epsilon}-u_{D}>d t+ \\
& +\int_{Q_{T}} a\left(\nabla u_{\epsilon}-\nabla v_{\epsilon}\right) \nabla u_{D}+\int_{Q_{T}} \epsilon \nabla u_{\epsilon} \nabla u_{D}-\int_{Q_{T}} a\left(\nabla u_{\epsilon}-\nabla v_{\epsilon}\right) \nabla v_{D}+\int_{Q_{T}} b \nabla v_{\epsilon} \nabla v_{D}
\end{aligned}
$$

En utilisant l'inégalité de Young, nous obtenons,

$$
\begin{aligned}
& \int_{Q_{T}} a\left|\nabla v_{\epsilon}-\nabla u_{\epsilon}\right|^{2}+\int_{Q_{T}} b\left|\nabla v_{\epsilon}\right|^{2}+\int_{Q_{T}} \epsilon\left|\nabla u_{\epsilon}\right|^{2} \leqslant \int_{0}^{T}<\frac{\partial \theta}{\partial t}\left(u_{\epsilon}\right), u_{\epsilon}-u_{D}>d t+ \\
& +\int_{Q_{T}} \frac{a}{4}\left|\nabla u_{\epsilon}-\nabla v_{\epsilon}\right|^{2}+\int_{Q_{T}} a\left|\nabla u_{D}\right|^{2}+\frac{\epsilon}{2} \int_{Q_{T}}\left|\nabla u_{\epsilon}\right|^{2}+\frac{\epsilon}{2} \int_{Q_{T}}\left|\nabla u_{D}\right|^{2}+ \\
& +\int_{Q_{T}} \frac{a}{4}\left|\nabla u_{\epsilon}-\nabla v_{\epsilon}\right|^{2}+\int_{Q_{T}} a\left|\nabla v_{D}\right|^{2}+\frac{1}{2} \int_{Q_{T}} b\left|\nabla v_{\epsilon}\right|^{2}+\frac{1}{2} \int_{Q_{T}} b\left|\nabla v_{D}\right|^{2}
\end{aligned}
$$

on trouve alors,

$$
\begin{aligned}
& \frac{1}{2} \int_{Q_{T}} a\left|\nabla v_{\epsilon}-\nabla u_{\epsilon}\right|^{2}+\frac{1}{2} \int_{Q_{T}} b\left|\nabla v_{\epsilon}\right|^{2}+\frac{1}{2} \int_{Q_{T}} \epsilon\left|\nabla u_{\epsilon}\right|^{2} \leqslant \int_{0}^{T}<\frac{\partial \theta}{\partial t}\left(u_{\epsilon}\right), u_{\epsilon}-u_{D}>d t+ \\
& \quad+\int_{Q_{T}} a\left|\nabla u_{D}\right|^{2}+\frac{\epsilon}{2} \int_{Q_{T}}\left|\nabla u_{D}\right|^{2}+\int_{Q_{T}} a\left|\nabla v_{D}\right|^{2}+\frac{1}{2} \int_{Q_{T}} b\left|\nabla v_{D}\right|^{2}
\end{aligned}
$$


D'après le lemme 3.3, nous avons la majoration: $\int_{0}^{T}<\frac{\partial \theta}{\partial t}\left(u_{\epsilon}\right), u_{\epsilon}-u_{D}>$ $d t \leqslant C$. En outre, les fonctions $a$ et $b$ sont dans $L^{\infty}\left(Q_{T}\right)$. Par conséquent, nous avons,

$$
\frac{1}{2} \int_{0}^{T} \int_{\Omega} a\left|\nabla v_{\epsilon}-\nabla u_{\epsilon}\right|^{2}+\frac{1}{2} \int_{0}^{T} \int_{\Omega} b\left|\nabla v_{\epsilon}\right|^{2}+\frac{1}{2} \int_{0}^{T} \int_{\Omega} \epsilon\left|\nabla u_{\epsilon}\right|^{2} \leqslant C
$$

et il vient alors,

$$
\begin{aligned}
\left\|\sqrt{\epsilon} u_{\epsilon}\right\|_{L^{2}(0, T ; V)} & \leqslant C, \\
\int_{0}^{T} \int_{\Omega} k_{w}\left(\theta\left(u_{\epsilon}\right)\right)\left|\nabla v_{\epsilon}-\nabla u_{\epsilon}\right|^{2} d x d t & \leqslant C, \\
\int_{0}^{T} \int_{\Omega} k_{a}\left(\theta\left(u_{\epsilon}\right)\right)\left|\nabla v_{\epsilon}\right|^{2} d x d t & \leqslant C
\end{aligned}
$$

D'après le principe du maximum, $\delta \leqslant u_{\epsilon} \leqslant u_{M}$, p.p. dans $Q_{T}$, et nous avons

$$
\alpha_{a *} \leqslant k_{a}(\theta(u)) \leqslant \alpha_{a}^{*}, \quad \forall u \in\left[\delta, u_{M}\right]
$$

où $\alpha_{a *}=k_{a}(\theta(\delta))$ et $\alpha_{a}^{*}=\sup _{t \in\left[\theta_{r}, \theta_{s}\right]}\left|k_{a}(\theta(t))\right|$.

Nous déduisons donc d'après (3.6) que,

$$
\left\|\nabla v_{\epsilon}\right\|_{L^{2}\left(0, T ; L^{2}(\Omega)\right)} \leqslant C
$$

et par conséquent,

$$
\begin{array}{r}
\int_{0}^{T} \int_{\Omega}\left|\nabla \phi\left(u_{\epsilon}\right)\right|^{2} d x d t=\int_{0}^{T} \int_{\Omega} k_{w}\left(\theta\left(u_{\epsilon}\right)\right)\left|\nabla u_{\epsilon}\right|^{2} d x d t \\
\leqslant 2 \int_{0}^{T} \int_{\Omega} k_{w}\left(\theta\left(u_{\epsilon}\right)\right)\left|\nabla u_{\epsilon}-\nabla v_{\epsilon}\right|^{2} d x d t+2 \int_{0}^{T} \int_{\Omega} k_{w}\left(\theta\left(u_{\epsilon}\right)\right)\left|\nabla v_{\epsilon}\right|^{2} d x d t \\
\leqslant 2 . C+2 . k_{w}^{*} \int_{0}^{T} \int_{\Omega}\left|\nabla v_{\epsilon}\right|^{2} d x d t \\
\leqslant C^{\prime}
\end{array}
$$

Nous avons alors,

$$
\begin{array}{r}
\left\|v_{\epsilon}\right\|_{L^{2}(0, T ; V)} \leqslant C \\
\left\|\nabla \phi\left(u_{\epsilon}\right)\right\|_{L^{2}\left(0, T ; L^{2}(\Omega)\right)} \leqslant C
\end{array}
$$

Prenons $\varphi \in L^{2}(0, T ; V)$ dans $(2.5)$, il vient,

$$
\begin{aligned}
-\int_{0}^{T}<\frac{\partial \theta}{\partial t}\left(u_{\epsilon}\right), \varphi>d t & +\int_{0}^{T} \int_{\Omega} a\left(\nabla u_{\epsilon}-\nabla v_{\epsilon}\right) \nabla \varphi d x d t=0 \\
& -544-
\end{aligned}
$$


ce qui implique,

$$
\begin{aligned}
\int_{0}^{T}<\frac{\partial \theta}{\partial t} & \left(u_{\epsilon}\right), \varphi>d t \\
& \left.\leqslant\left. C\left(\int_{0}^{T} \int_{\Omega} a \mid \nabla u_{\epsilon}-\nabla v_{\epsilon}\right)\right|^{2} d x d t\right)^{1 / 2} \cdot\left(\int_{0}^{T} \int_{\Omega}|\nabla \varphi|^{2} d x d t\right)^{1 / 2}
\end{aligned}
$$

En vertu de (3.5), on déduit que,

$$
\int_{0}^{T}<\frac{\partial \theta}{\partial t}\left(u_{\epsilon}\right), \varphi>d t \leqslant C\|\varphi\|_{L^{2}(0, T ; V)}
$$

ce qui conduit à l'estimation,

$$
\left\|\frac{\partial \theta}{\partial t}\left(u_{\epsilon}\right)\right\|_{L^{2}\left(0, T ; V^{\prime}\right)} \leqslant C
$$

En utilisant le lemme 3.4 , nous obtenons,

$$
\begin{array}{r}
-\int_{0}^{T-h} \int_{\Omega}\left(\theta\left(u_{\epsilon}(t+h)\right)-\theta\left(u_{\epsilon}(t)\right)\right)\left(\phi\left(u_{\epsilon}(t+h)\right)-\phi\left(u_{\epsilon}(t)\right)\right) d x d t \leqslant 2 C . h, \\
\forall h \in] 0, T[
\end{array}
$$

où $C$ est indépendante de $\epsilon$.

En remarquant que $\theta\left(u_{\epsilon}\right)=\left(\theta o \phi^{-1}\right)\left(\phi\left(u_{\epsilon}\right)\right)$ et d'après le lemme 2.5, nous déduisons qu'il existe $\chi_{1} \in L^{1}\left(Q_{T}\right)$ telle que:

$$
\theta\left(u_{\epsilon}\right) \rightarrow \chi_{1}, \quad \text { dans } L^{1}\left(Q_{T}\right) \text { fort et p.p. dans } Q_{T} .
$$

Puisque $\theta$ est inversible et $\theta^{-1}$ est continue, et en vertu du théorème de Lebesgue, nous obtenons,

$$
\begin{gathered}
u_{\epsilon} \rightarrow u=\theta^{-1}\left(\chi_{1}\right) \quad \text { dans } L^{p}\left(Q_{T}\right) \text { fort, } \forall p \in\left[1, \infty\left[\text {, et p.p. dans } Q_{T}\right.\right. \\
\phi\left(u_{\epsilon}\right) \rightarrow \phi(u) \quad \text { dans } L^{p}\left(Q_{T}\right) \text { fort, } \forall p \in\left[1, \infty\left[\text {, et p.p. dans } Q_{T}\right.\right.
\end{gathered}
$$

D'après les estimations (3.7)-(3.9), on en déduit que,

$$
\exists v \in L^{2}\left(0, T ; H^{1}(\Omega)\right): \quad v_{\epsilon} \rightarrow v, \quad \text { dans } L^{2}(0, T ; V) \text { faible }
$$

$$
\begin{aligned}
\exists \chi_{1}^{\prime} \in L^{2}\left(0, T ; H^{1}(\Omega)\right): \quad \phi\left(u_{\epsilon}\right) & \rightarrow \chi_{1}^{\prime}, \quad \text { dans } L^{2}(0, T ; V) \text { faible } \\
\exists \chi_{2} \in L^{2}\left(0, T ; V^{\prime}\right): \quad \frac{\partial \theta\left(u_{\epsilon}\right)}{\partial t} & \rightarrow \chi_{2}, \quad \text { dans } L^{2}\left(0, T ; V^{\prime}\right) \text { faible } \\
& -545-
\end{aligned}
$$


Par identification des limites, nous obtenons tout d'abord $\chi_{1}^{\prime}=\phi(u)$, puis nous obtenons, en vertu de (3.10),

$$
\chi_{2}=\frac{\partial \theta(u)}{\partial t}
$$

En vertu des estimations (3.4) et (3.11)-(3.13), nous pouvons passer à la limite dans (2.4)-(2.6) pour obtenir que $(u, v)$ est solution du problème dégénéré.

Remerciements. - Les auteurs remercient B. Amaziane et G. Vallet du Laboratoire de Mathématiques appliquées de l'Université de Pau et des Pays de L'Adour pour leurs suggestions et remarques fructueuses le long de ce travail.

\section{Bibliographie}

[1] Alt (H. W.) and Luckhauss (S.). - Quasilinear elliptic-parabolic equations, Math. Z. 183, pp. 311-341, 1983.

[2] Amaziane (B.), Bourgeat (A.) et El Amri (H.). - Un résultat d'existence pour un modèle d'écoulement diphasique dans un milieu poreux à plusieurs types de roches. Publication du Laboratoire d'Analyse Numérique No. 90-17, Université de Pau, 1990.

[3] Amirat (Y.), Gabbouhy (M.) et Mghazli (Z.). - Ecoulement diphasique dans un milieu poreux non saturé, Rapport No. R1/96, Laboratoire SIANO, Université Ibn Tofaïl, Kénitra, Maroc.

[4] Antonsev (S. N.), Kazhikhov (A. V.) and Monakhov (V. N.). - Boundary value problems in mechanics of nonhomogeneous fluids. Studies in mathematics and its applications, Vol. 22, North-Holland, Amsterdam, 1990.

[5] Arbogast (T.). - The existence of weak solutions to single porosity and simple dual-porosity models of two-phase incompressible flow, Nonlinear Analysis, Theory, Methods and Applications, Vol. 19, No. 11, pp. 1009-1031, 1992.

[6] BrÉzis (H.). - Analyse fonctionnelle, Théorie et application. Masson, 1983.

[7] Chavent (G.) and Jaffré (J.). - Mathematical models and finite elements for reservoir simulation. Studies in mathematics and its applications, Vol. 17, North-Holland, Amsterdam, 1986.

[8] Gagneux (G.) et Madaune-Tort (M.). - Analyse mathématique de modèles non linéaires de l'ingénierie pétrolière. Springer 1996.

[9] Hidani (A.). - Modélisation des écoulements diphasiques en milieux poreux à plusieurs types de roches. Thèse de Doctorat, Université de Saint-Etienne, 1993.

[10] Kroener (D.) and Luckhauss (S.). - Flow of oil and water in a porous medium. J. of Differential Equations 55, pp. 276-288, 1984.

[11] Touma (J.) and VAuclin (M.). - Experimental and numerical analysis of two-phase infiltration in a partially saturated soil, Transport in Porous Media, 1, pp. 27-55, 1986. 